\title{
Manfred Grauer
}

\section{Einführung}

Gegenstand der Beiträge dieser Sektion waren die Wechselwirkungen zwischen Medienwissenschaften und Informatik. Dabei ging es um die durch die Informationstechnik neu geschaffenen (Peter Gendolla, Universität Siegen) und zu erschaffenden Medien (Manfred Jarke, RWTH Aachen, Volker Wulf, Universität Siegen, Sigrid Schubert, Universität Siegen). Daneben wurde kritisch die Frage erörtert, wie sich die Medienwissenschaften zukünftig entwickeln sollten (Wolfgang Coy, Humboldt-Universität Berlin).

Der gegenwärtige Medienumbruch stellt in Bezug auf die Medien eine bedeutende Veränderung dar. Durch die neuen Möglichkeiten einer »Rückkopplung « zwischen Medien-Produzent und Medien-Konsument lösen sich hier bestehende Grenzen auf. Im Internet kann jeder Sender und Empfänger sein und mit jedem kommunizieren. Die Ebenen zwischen auf Einzelpersonen bezogener Kommunikation und Massenkommunikation gehen dabei fließend ineinander über.

Aus ökonomischer Sicht sind bei digitalen Medien als immaterielle Produkte andere Eigenschaften als bei materiellen Produkten dominant. Die Kosten für die erstmalige Erstellung eines digitalen Produkts (Musik, Film etc.) sind in der Regel sehr hoch. Jedoch die Kosten für die Erstellung jeder weiteren Kopie sind vernachlässigbar gering, so dass die Erträge eines immateriellen Produkts steigen, je mehr von diesem Produkt vertrieben wird. Diese Eigenschaften der so genannten »Positiven Rückkopplungseffekte (increasing returns)« führen zu Konsequenzen, deren ökonomische, technologische und juristische Aspekte in den Wirtschaftswissenschaften, Rechtswissenschaften und der Informatik untersucht werden. Für die Betrachtung der kulturellen, sozialen und ästhetischen Konsequenzen bedarf es der Medienwissenschaften.

Peter Gendolla beschreibt in seinem Beitrag die tiefgreifenden Veränderungen der Medienwissenschaften, die sich durch die Informationstechnik ergeben. Er führt diese auf die Entstehung neuer Medien durch Digitalisierung und Integration bisheriger Medien in rechnergestützten und vernetzten Systemen zurück. Als Merkmal dieser neuen Medien macht er ihre ständige Veränderbarkeit und Zusammensetzung aus modularen Teilen aus.

Mathias Jarke berichtet vom SFB/FK 427 Köln-Aachen-Bonn »Medien und Kulturelle Kommunikation « in seinem Beitrag zum Thema »Transkriptivität als informatorisches Designprinzip«. An Hand der Ergebnisse der ersten Projektphase wird darin verdeutlicht, wie sich durch die Erstellung einer 
Medienstruktur Auswirkungen auf den Adressatenkreis des Inhalts ergaben. Ein weiteres Ergebnis betrifft die Untersuchung des unterschiedlichen Begriffsgebrauchs zwischen ingenieur- und kulturwissenschaftlichen Projekten. Im aktuellen Projekt wird die Auswirkung von e-learning-Techniken auf spezielle communities untersucht.

Im Beitrag von Volker Wulf wird die Medieninformatik als Teil der angewandten Informatik beschrieben. Es wird erläutert, inwieweit für die Gestaltung digitaler Medien geistes- und sozialwissenschaftliche Theorien und Methoden benötigt werden.

Sigrid Schubert setzt sich kritisch mit den Möglichkeiten der Informatik im Bereich des E-learnings auseinander. Sie berichtet vom BMBF-Verbundprojekt »Simba«, welches Lehrende bei der Vermittlung von Schlüsselkonzepten durch einzelne E-learning-Bausteine unterstützt.

Wolfgang Coy diskutiert schließlich die Frage der »flächendeckenden« Einführung von Medienwissenschaft als universitärer Disziplin. Der flächendeckenden Gründung wird dabei widersprochen. Dies wird mit der generellen Einführung der Bachelor- und Masterstudiengänge und der damit zunehmenden Modularisierung begründet. Andererseits sieht Coy die Existenzberechtigung eines Forschungsgebietes »Medienwissenschaften« durch erfolgreiche Praxis an herausragenden Standorten für geklärt. 